\title{
Anlotinib is effective in the treatment of advanced carcinoma ex pleomorphic adenoma of the submandibular gland
}

This article was published in the following Dove Press journal: OncoTargets and Therapy

\author{
Shujie Song* \\ Ping Sui* \\ Minmin Li \\ Liangming Zhang \\ Dengjun Sun
}

Department of Medical Oncology, The Affiliated Yantai Yuhuangding Hospital of Qingdao University, Yantai, Shandong,

People's Republic of China

*These authors contributed equally to this work
Correspondence: Dengjun Sun Department of Medical Oncology, The Affiliated Yantai Yuhuangding Hospital of Qingdao University, Yantai, Shandong 264000, People's Republic of China

Tel +860535669199981903

Fax +8605356691999

Email sundengjun@sina.com
Background: Carcinoma ex pleomorphic adenoma (CXPA), a very rare malignancy found mostly in the major salivary glands, has no established standardized treatment.

Case presentation: This report describes a 67-year-old male with advanced CXPA who was effectively treated by anlotinib. Pleomorphic adenoma of the submandibular gland was first diagnosed in 1976 after a surgical resection of a mass underneath the jaw. The patient underwent re-excision 3 years later due to a recurrent pleomorphic adenoma. CXPA was first diagnosed in 2016 after a surgical removal of the left submandibular mass. A lung nodule was found on a chest CT scan in January 2018. Following a CTguided lung biopsy that demonstrated findings consistent with pulmonary metastasis, the patient underwent local therapy (microwave ablation and radioactive seed implantation) but suffered a recurrence of disease approximately 6 months later. Anlotinib was administered orally at a dose of $12 \mathrm{mg}$ daily on a 2 weeks on/1 week off schedule. A partial response was observed after two cycles of treatment. The disease remains in continued partial response after completion of his sixth cycle.

Conclusion: This is the first report for anlotinib in treating CXPA. Further pre-clinical and clinical studies are needed to validate the efficacy and safety of anlotinib in the treatment of CXPA.

Keywords: pulmonary metastasis, chemotherapy, partial response, angiogenesis

\section{Introduction}

Salivary gland tumors are a morphologically and clinically diverse group of rare neoplasms originating in the major glands (parotid, submandibular, and sublingual) and minor glands (eg, oral mucosa, palate, uvula, pharynx, larynx, and paranasal sinuses). The most common type of benign salivary gland tumors is pleomorphic adenoma, which has a malignant potentiality. Malignant pleomorphic adenoma is a rare salivary gland neoplasm that is histologically identical to pleomorphic adenoma that inexplicably metastasize. ${ }^{1}$ According to the 2005 third histologic classification of the World Health Organization, there are three distinct clinicopathologic types for malignant pleomorphic adenoma: carcinoma ex pleomorphic adenoma (CXPA), carcinosarcoma, and metastasizing mixed tumor. CXPA is one of the three types of malignant pleomorphic adenoma arising from a primary or recurrent benign pleomorphic adenoma. The disease is very rare, for it has a prevalence rate of 5.6 cases per 100,000 malignant neoplasms and a yearly incidence rate of 0.17 tumors per 1 million 
persons. ${ }^{2}$ The cancer is found predominantly in the parotid and submandibular glands.

No standardized treatment guideline is available for CXPA. Current treatment modalities consist of surgical resection, chemotherapy and radiotherapy. No targeted therapy options have been established for CXPA.

Anlotinib (Jiangsu Chia-tai Tianqing Pharmaceutical Co., Ltd, People'sRepublic of China) is an oral receptor tyrosine kinase (RTK) inhibitor and targets multiple RTKs including vascular endothelial growth factor receptor type (VEGFR)1 to 3, EGFR, platelet-derived growth factor receptor $\alpha$ and $\beta$, and fibroblast growth factor receptor (FGFR) 1-3. ${ }^{3,4}$ Anlotinib has been proved to be effective and safe as a third line treatment in patients with refractory advanced non-small cell lung cancer. ${ }^{5}$ There are also ongoing Phase I/II clinical trials for different types of sarcomas and carcinomas in China and other countries. However, to the best of our knowledge, no studies have been reported for anlotinib in treating CXPA. Here we report a case of recurrent CXPA which was effectively treated by anlotinib.

Written informed consent was obtained from the patient. Institutional approval was not required to publish the case details.

\section{Case report Clinical presentation}

On July 8, 2016, a 67-year-old Chinese male was admitted to Jinan military general hospital due to a mass underneath the jaw. Neither numbness nor facial nerve weakness was reported. The patient received submandibular gland surgery in 1976 and 1979, respectively, to resect pleomorphic adenoma. Physical examination revealed that the mass had hard texture, unclear boundary, and a low degree of mobility. The patient's treatment included removal of the left submandibular gland and concomitant neck dissection. Immunohistochemistry (IHC) revealed that tumor cells were positive to GFAP, CK5/6, S-100, CK8/CK18, and negative for $\mathrm{CD} 117$ and $\mathrm{p} 63$. Ki-67 proliferation index was $30-40 \%$. (Figure $1 \mathrm{~A}-\mathrm{C}$ ) No metastasis to the lymph nodes in the neck was found. The pathologic findings were supportive for the diagnosis of CXPA with a carcinomatous component of adenocarcinoma. Postoperative radiation therapy was carried out to target the operative bed (60 Gy in 30 fractions).

Fourteen months ago, the patient was re-hospitalized due to a lung nodule found on a chest CT scan. A CT-guided lung needle biopsy was performed. Histopathological

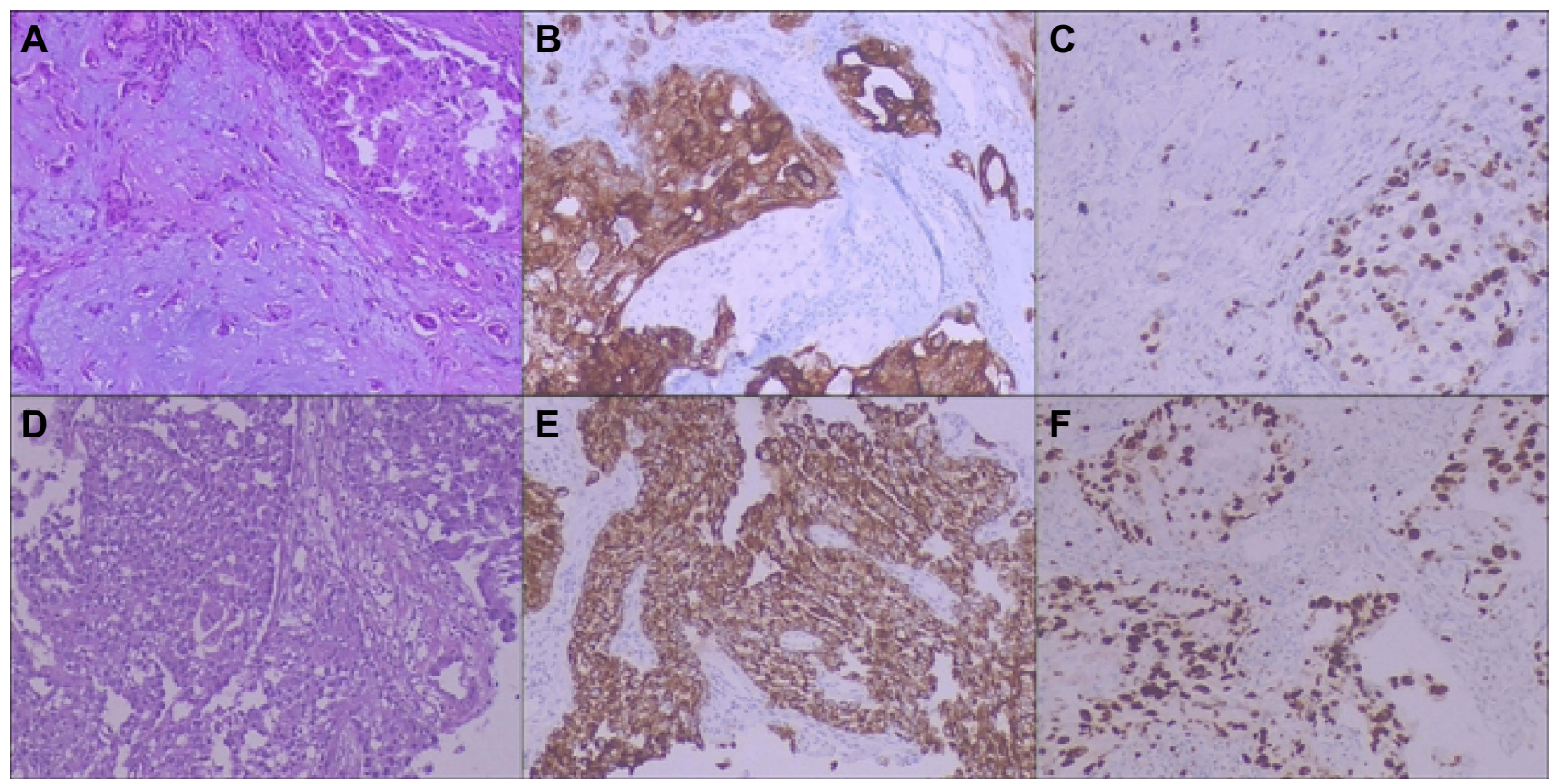

Figure I (A) Histology of primary carcinoma ex pleomorphic adenoma; (B) Immunohistochemistry showed that tumor cells were positive for CK8/I8. (C) Immunohistochemistry showed that Ki 67 was 30-40\%. (D) Histology of pulmonary metastasis. (E) Immunohistochemistry showed that tumor cells were positive for CK8/I8. (F) Immunohistochemistry showed that Ki 67 was about $70 \%$. (A-F) Original magnification, $\times 100$, (A-C: primary carcinoma ex pleomorphic adenoma of the submandibular gland, D-F: pulmonary metastasis, A and D: hematoxylin-eosin). 
examination revealed findings consistent with metastatic CXPA with adenocarcinoma component. On IHC, the tumor biopsy was positive for $\mathrm{CK} 7, \mathrm{CK} 8 / \mathrm{CK} 18$, weak positive for villin, and negative for CK20, TTF-1, Napsin A, CK5/CK6 and p63. Ki67 was about 70\% (Figure 1D-F). The patient underwent microwave ablation and radioactive seed implantation, respectively, but suffered a recurrence of disease approximately 6 months later. Anlotinib was administered orally at a dose of $12 \mathrm{mg}$ daily on days $1-14$ of a 21 day cycle. ${ }^{4}$ As shown in Figure 2, partial response was observed after two cycles of treatment. The disease remains in sustained partial response after completion of his sixth cycle.

\section{Discussion}

CXPA typically presents as a firm mass in the parotid or submandibular gland. It is very difficult to diagnose preoperatively. About a quarter of patients had a previously treated salivary pleomorphic adenoma. ${ }^{6,7}$ Treatment for CXPA remains challenging because conducting large randomized clinical trials is not realistic due to the entity's low incidence. The major approach is surgical resection and radiotherapy. The value of chemotherapy is inclusive in advanced disease. There is very scant literature on chemotherapy in the treatment of CXPA. Several agents alone or in combination (vinorelbine, paclitaxel, cyclophosphamide, doxorubicin, and cisplatin) have been shown to be effective for some salivary gland malignancies. ${ }^{8-12}$ In a Phase II randomized trial comparing vinorelbine versus vinorelbine plus cisplatin in patients with recurrent salivary gland malignancies, no response was observed in the patient with a malignant pleomorphic adenoma. ${ }^{8}$ In 2009 , Lüers JC et al ${ }^{13}$ retrospectively analyzed 22 patients with CXPA of the parotid gland and showed that patients receiving chemotherapy even had a lower 5-year overall survival rate $(50 \%)$ than those receiving no chemotherapy (63\%). However, in a case report of CXPA from a Canadian group, chemotherapy with CAP (cyclophosphamide, doxorubicin, and cisplatin) result in an excellent clinical and radiologic response. ${ }^{14}$ Therefore, the role of chemotherapy in CXPA is still unclear. Due to the low number of patients, it is very difficult to conduct high-quality clinical trials for the disease. No targeted therapy options have been established for CXPA. Two case reports reported durable responses to treatment with trastuzumab in combination with chemotherapy in epidermal growth factor receptor 2 (HER2) positive patients. ${ }^{15,16}$ However, clinical trials of HER2 targeted therapies in malignant salivary tumors has been disappointing. In a Phase II study of HER2 positive advanced salivary gland cancers, the overall response to trastuzumab was only $7.1 \%(1 / 14) .{ }^{17}$ Another Phase II study of lapatinib showed no objective response in 36 patients. $^{18}$

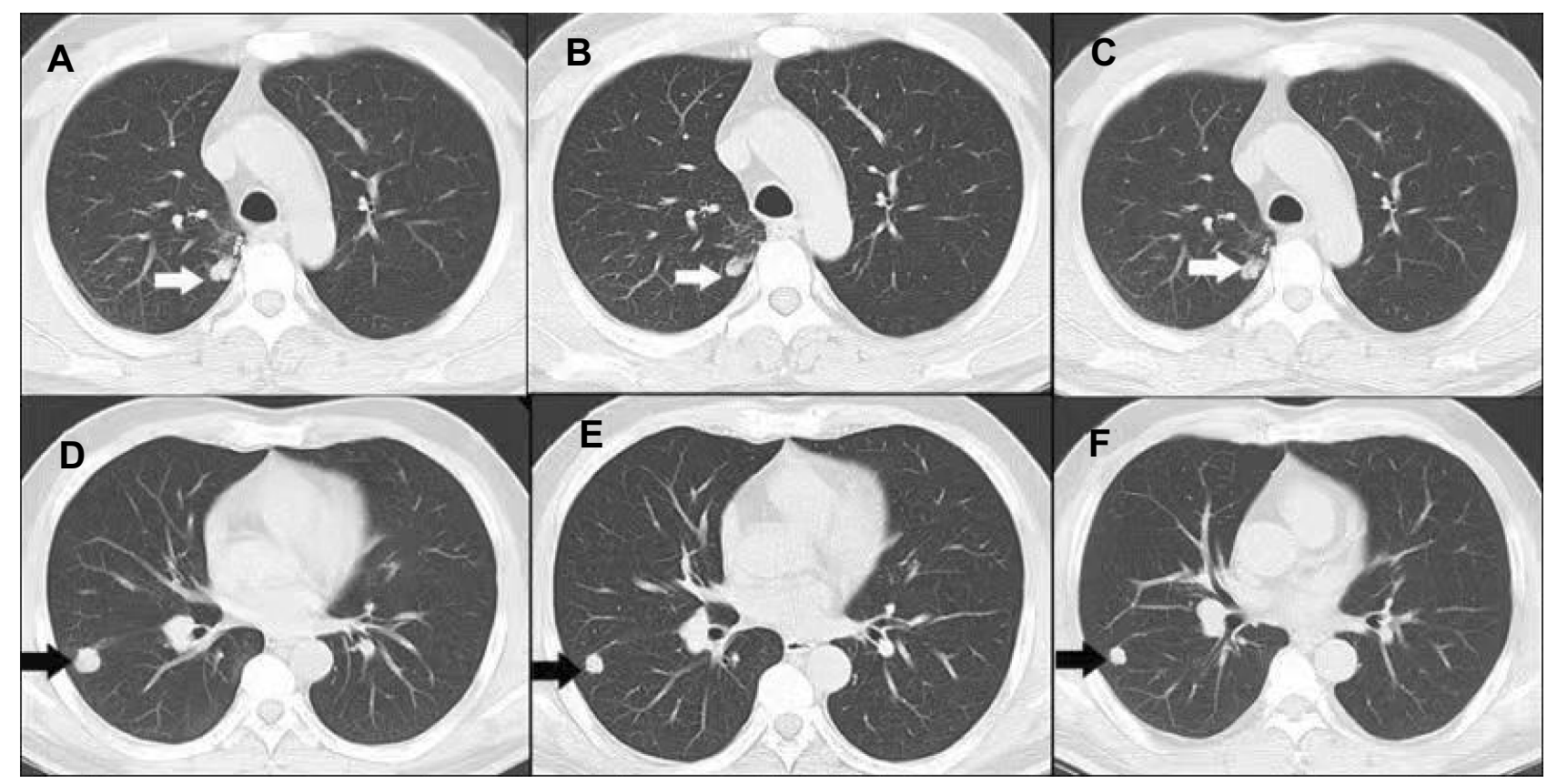

Figure 2 Chest CT scans before and after anlotinib treatment. (A and $\mathbf{D}$ ): Before anlotinib treatment, two measurable tumor lesions were shown. (B and $\mathbf{E})$ After two cycles of anlotinib treatment, a Partial response was observed. (C and F) After six cycles of anlotinib treatment, a continued partial response was observed. The white and black arrowheads aim at two measurable tumor lesions, respectively. Response assessment was based on RECIST guideline version I.I. 
Several genes associated with tumor angiogenesis, such as $H G F-A, M E T$ and $C O X 2$, were found to be highly expressed in CXPA. ${ }^{19,20}$ FGFR1-3, IGFIR, $P D G F R \quad \beta$ and EGFR were also reported to be overexpressed in CXPA. ${ }^{14,20,21}$ Anlotinib has a potent inhibitory action on tumor angiogenesis and was reported to target VEGFR1 to 3, EGFR, MET, PDGFR $\alpha$ and $\beta$, and FGFR $1-4 .{ }^{4}$ Based on potential targetable pathways identified above, a multi-targeted tyrosine kinase inhibitor, like anlotinib, was a rational option for advanced CXPA.

In this case, the patient developed pulmonary metastasis 42 years after the first surgery. His disease progressed approximately 6 months after local therapy. Anlotinib was selected and resulted in an excellent response. No severe toxicity except grade 1 nausea was observed in the patient.

To the best of our knowledge, this is the first report for anlotinib in treating CXPA. Further pre-clinical and clinical studies are needed to validate the efficacy and safety of anlotinib in the treatment of CXPA.

\section{Acknowledgments}

We would like to acknowledge Dr Darmood Wei at the National Cancer Institute (USA) for providing his editorial expertise in this manuscript. This study was funded by Shandong Natural Science Foundation (Grant number: ZR2016HL28).

\section{Disclosure}

The authors report no conflicts of interest in this work.

\section{References}

1. Wenig BM, Hitchcock CL, Ellis GL, Gnepp DR. Metastasizing mixed tumor of salivary glands. A clinicopathologic and flow cytometric analysis. Am J Surg Pathol. 1992;16(9):845-858.

2. Antony J, Gopalan V, Smith RA, Lam AKY. Carcinoma ex pleomorphic adenoma: a comprehensive review of clinical, pathological and molecular data. Head Neck Pathol. 2011;6(1):1-9. doi:10.1007/ s12105-011-0281-z

3. Lin B, Song X, Yang D, Bai D, Yao Y, Lu N. Anlotinib inhibits angiogenesis via suppressing the activation of VEGFR2, PDGFRbeta and FGFR1. Gene. 2018;654:77-86. doi:10.1016/j. gene.2018.02.026

4. Sun Y, Niu W, Du F, et al. Safety, pharmacokinetics, and antitumor properties of anlotinib, an oral multi-target tyrosine kinase inhibitor, in patients with advanced refractory solid tumors. J Hematol Oncol. 2016;9(1):105. doi:10.1186/s13045-016-0332-8
5. Han B, Li K, Zhao Y, et al. Anlotinib as a third-line therapy in patients with refractory advanced non-small-cell lung cancer: a multicentre, randomised phase II trial (ALTER0302). $\mathrm{Br}$ J Cancer. 2018;118(5):654-661. doi:10.1038/bjc.2017.478

6. Nouraei SA, Hope KL, Kelly CG, McLean NR, Soames JV. Carcinoma ex benign pleomorphic adenoma of the parotid gland. Plast Reconstr Surg. 2005;116(5):1206-1213.

7. Zbaren P, Zbaren S, Caversaccio MD, Stauffer E. Carcinoma ex pleomorphic adenoma: diagnostic difficulty and outcome. Otolaryngol-Head Neck Surg. 2008;138(5):601-605. doi:10.1016/j. otohns.2008.01.013

8. Airoldi M, Pedani F, Succo G, et al. Phase II randomized trial comparing vinorelbine versus vinorelbine plus cisplatin in patients with recurrent salivary gland malignancies. Cancer. 2001;91(3):541-547.

9. Licitra L, Cavina R, Grandi C, et al. Cisplatin, doxorubicin and cyclophosphamide in advanced salivary gland carcinoma. A phase II trial of 22 patients. Ann Oncol. 1996;7(6):640-642.

10. Laurie SA, Licitra L. Systemic therapy in the palliative management of advanced salivary gland cancers. J Clin Oncol. 2006;24 (17):2673-2678. doi:10.1200/JCO.2005.05.3025

11. Gilbert J, Li Y, Pinto HA, et al. Phase II trial of taxol in salivary gland malignancies (E1394): a trial of the Eastern Cooperative Oncology Group. Head Neck. 2006;28(3):197-204. doi:10.1002/hed.20327

12. Debaere D, Vander Poorten V, Nuyts S, et al. Cyclophosphamide, doxorubicin, and cisplatin in advanced salivary gland cancer. B-Ent. 2011;7 (1): $1-6$.

13. Luers JC, Wittekindt C, Streppel M, Guntinas-Lichius O. Carcinoma ex pleomorphic adenoma of the parotid gland. Study and implications for diagnostics and therapy. Acta Oncol. 2009;48(1):132-136. doi: $10.1080 / 02841860802183604$

14. Chooback N, Shen Y, Jones M, et al. Carcinoma ex pleomorphic adenoma: case report and options for systemic therapy. Curr Oncol. 2017;24(3):e251-e254. doi:10.3747/co.24.3588

15. Sharon E, Kelly RJ, Szabo E. Sustained response of carcinoma ex pleomorphic adenoma treated with trastuzumab and capecitabine. Head Neck Oncol. 2010;2:12. doi:10.1186/1758-3284-2-12

16. Kadowaki S, Yatabe Y, Hirakawa H, et al. Complete response to trastuzumab-based chemotherapy in a patient with human epidermal growth factor receptor-2-positive metastatic salivary duct carcinoma ex pleomorphic adenoma. Case Rep Oncol. 2013;6(3):450-455. doi:10.1159/000355219

17. Haddad R, Colevas AD, Krane JF, et al. Herceptin in patients with advanced or metastatic salivary gland carcinomas. A phase II study. Oral Oncol. 2003;39(7):724-727.

18. Agulnik M, Cohen EW, Cohen RB, et al. Phase II study of lapatinib in recurrent or metastatic epidermal growth factor receptor and/or erbB2 expressing adenoid cystic carcinoma and non adenoid cystic carcinoma malignant tumors of the salivary glands. J Clin Oncol. 2007;25(25):3978-3984. doi:10.1200/JCO.2007.11.8612

19. Katori H, Nozawa A, Tsukuda M. Increased expression of cyclooxygenase- 2 and Ki-67 are associated with malignant transformation of pleomorphic adenoma. Auris Nasus Larynx. 2007;34 (1):79-84. doi:10.1016/j.anl.2006.09.006

20. Furuse C, Miguita L, Rosa AC, et al. Study of growth factors and receptors in carcinoma ex pleomorphic adenoma. J Oral Pathol Med. 2010;39(7):540-547.

21. Katori H, Nozawa A, Tsukuda M. Expression of epidermal growth factor receptor, transforming growth factor-alpha and $\mathrm{Ki}-67$ in relationship to malignant transformation of pleomorphic adenoma. Acta Otolaryngol. 2007;127(11):1207-1213. doi:10.108 0/00016480701230894 


\section{Publish your work in this journal}

OncoTargets and Therapy is an international, peer-reviewed, open access journal focusing on the pathological basis of all cancers, potential targets for therapy and treatment protocols employed to improve the management of cancer patients. The journal also focuses on the impact of management programs and new therapeutic agents and protocols on patient perspectives such as quality of life, adherence and satisfaction. The manuscript management system is completely online and includes a very quick and fair peer-review system, which is all easy to use. Visit http://www.dovepress.com/ testimonials.php to read real quotes from published authors.

Submit your manuscript here: https://www.dovepress.com/oncotargets-and-therapy-journal 